\title{
Objeto, experiencia, conocimiento en la obra de arte
}

\section{Object, experience, knowledge in the artwork}

\author{
Víctor Viviescas Monsalve*
}

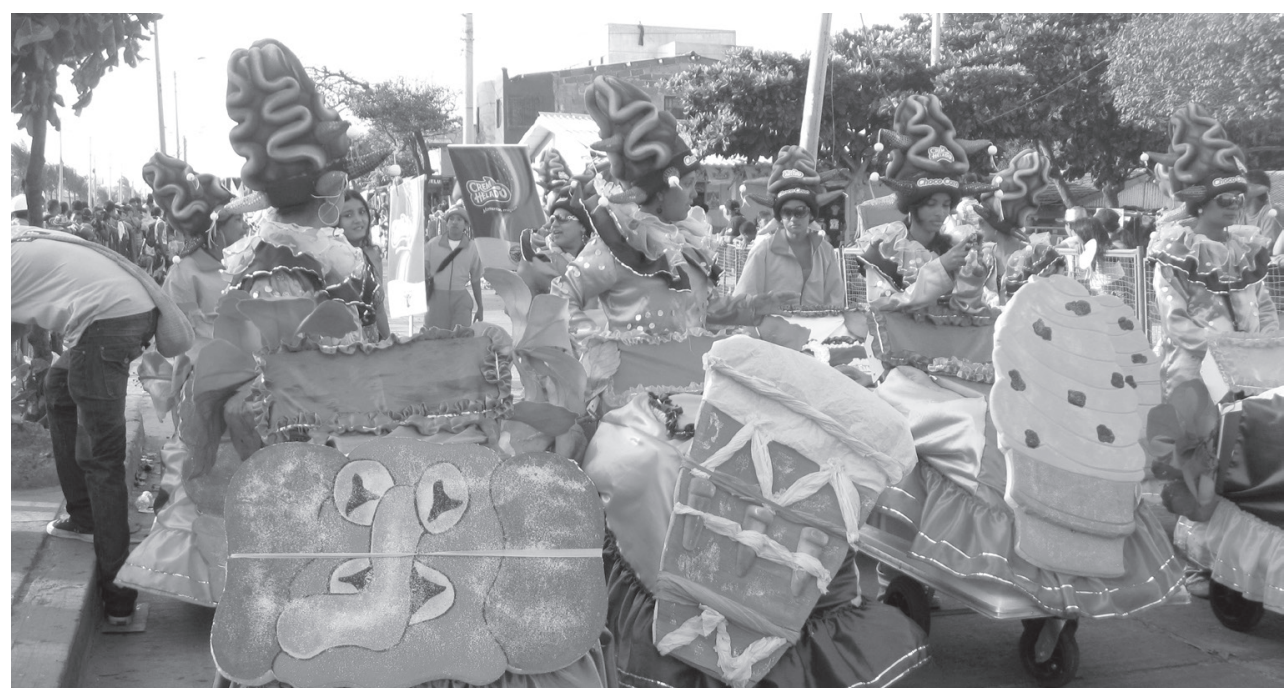

\section{Resumen}

Este texto se propone hacer un balance de la experiencia estética que produce la obra de arte pasando por sus momentos de objeto, experiencia y conocimiento. Aquí se postula que el conocimiento es el momento de síntesis de la experiencia estética, en la que el momento inefable de la obra de arte se manifiesta como conocimiento otro (otro, sin duda, respecto del conocimiento discursivo y conceptual). En esta consideración, la obra de arte es interrogada respecto de la estética, pero de manera especial en la comprensión que aportan las reflexiones de G. W. F. Hegel y Theodor Adorno, en una línea de pensamiento que desde mi propia indagación se inscribe en la problemática de la representación, de manera precisa, en la de políticas de la

Citar este artículo como: Viviescas Monsalve, V. (2017). Objeto, experiencia, conocimiento en la obra de arte. Revista Papeles, 9(18), 63-72.

Fecha de recibido: julio 1 de 2017.

Fecha de aceptación: octubre 30 de 2017.

* Dramaturgo, director e investigador teatral. Director de la Maestría en Escrituras Creativas de la Universidad Nacional de Colombia y director de Teatro Vreve. Sus obras han sido traducidas al francés y al portugués y publicadas en diferentes países de América y Europa. Viviescas es doctor en Estudios Teatrales de la Universidad Paris 3 y Magister en Literatura de la Universidad Javeriana de Bogotá. Como investigador en literatura ha sido coautor de Variaciones: seis ensayos de literatura comparada (2011), Representaciones, identidades y ficciones. Lectura crítica de las historias de la literatura latinoamericana (2010) y Topo/grafías. Literatura y región: el caso de Bogotá (2016). Integra el Grupo de Investigación Historia y Literatura y lidera el Grupo de Investigación en Estética e Historia del Teatro Colombiano y Latinoamericano de la misma universidad. Correo: vrviviescasm@unal.edu.co 
representación. Esta reflexión la realizo en el contexto de una discusión sobre la investigación-creación en el marco de los espacios de la academia, en especial, en las universidades que imparten enseñanza en el nivel profesional y posgradual en artes. Este contexto no puede perderse de vista, por más que nuestro arribo a él pueda ser un tanto moroso. En todo caso, el camino construye el lugar de llegada y nos deberá aportar los elementos que permitan una reflexión y la postulación de estrategias en el campo de la investigación-creación.

Palabras clave: experiencia, objeto, conocimiento, estética, políticas de la representación.

\begin{abstract}
This text aims to take stock of the aesthetic experience produced by the work of art through its moments of object, experience and knowledge. Here it is postulated that knowledge is the moment of synthesis of the aesthetic experience, in which the ineffable moment of the work of art manifests itself as another knowledge (another, no doubt, with respect to discursive and conceptual knowledge). In this regard, the work of art is questioned about aesthetics, but in a special way in the understanding provided by the reflections given by GWF Hegel and Theodor Adorno, in a line of thought that from my own inquiry is inscribed in the problematic of the representation, in a precise manner, in the politics of representation. I make this reflection in the context of a discussion about research-creation within the framework of academic spaces, especially in the universities that teach at the professional and postgraduate levels in the field of arts. This context cannot be lost sight of, even though our arrival to it may be a bit slow. In any case, the road constructs the place of arrival and must provide us with the elements that allow reflection and the postulation of strategies in the field of research-creation.
\end{abstract}

Key words: experience, object, knowledge, aesthetics, politics of representation.

\title{
La representación como disposición de lo real para la escritura
}

Suelo moverme tanto en el campo de la creación literaria -al menos como dimensión literaria de la escritura dramática- como en el de la acción teatral y performática. Encuentro que el concepto de "escritura" es lo bastante amplio como para abarcar procesos de creación en el campo estrictamente literario, tanto como en el campo de la creación escénica, actoral y performática. En todo caso, quiero relacionar la escritura como práctica de composición y conformación de los artefactos artísticos que se construyen con materiales verbales, tanto como aquellos que se construyen con materiales propiamente matéricos, si me permiten esta redundancia, de manera especial los que se construyen con el cuerpo. Pero en esta reflexión querría incluir en el arte las prácticas del sonido y del arte plástico. En estos campos también la composición puede ser asimilada a una operación de escritura.

Me apoyo en Theodor Adorno para hacer esta asimilación, de quien me apropio del sentido de la práctica de la escritura que él establece, al señalar la condición procesual de la obra de arte -asunto sobre el que volveremos más adelante-. Respecto de la escritura como procedimiento de conformación expansible a disciplinas no literarias, dice Theodor Adorno: 
"las obras de arte son escritura porque, como en los signos del lenguaje, su aspecto procesual se codifica en su objetivación" (Adorno, 2004, p. 237). Aquí Adorno destaca y valora la condición de devenir como signo de los materiales -incluso los que no son palabras-; yo destaco y pongo de relieve un segundo sentido que ya está allí en la cita: la dimensión composicional de la escritura. Ya en el aspecto procesual de la obra de arte que destaca Adorno se deja reconocer el momento de conformación-configuración. En efecto, la obra de arte no "es", sino que "llega a ser": es proceso, no substancia o esencia. Es el resultado de la configuración, de un devenir artefacto. Sigue siendo proceso, también, en el momento del encuentro con el espectador, lo que constituye propiamente la experiencia estética.

Theodor Adorno destaca entonces la condición procesual de la obra de arte, su constante devenir, en el proceso de construcción y en el momento del encuentro con el espectador. Esta condición procesual aparece, en parte, como consecuencia de su carácter polémico. El carácter polémico proviene de que la obra de arte es interrogadora o cuestionadora de la realidad que existe como tal: la obra de arte se afirma y en su afirmación pone en crisis el sentido de lo real tal como este existe: "Todas las obras de arte, incluso las afirmativas, son polémicas a priori. La idea de una obra de arte conservadora tiene algo de disparatado" (Adorno, 2004, p. 236).

Esta condición, no obstante, proviene de la relación que la obra de arte establece con la realidad. En su proceso de llegar a ser, la obra de arte se separa del mundo del que proviene, y reclama su transformación, es decir, expresa la inconformidad del mundo tal cual es:

Al separarse enfáticamente del mundo empírico, de su otro, las obras de arte proclaman que el mundo empírico tiene que cambiar: son esquemas inconscientes del cambio del mundo empírico. Incluso en artistas en apariencia no polémicos, que se mueven en una esfera del espíritu pura (según la convención), como Mozart, (...) el momento polémico es central, la fuerza del distanciamiento que condena sin palabras lo mezquino y lo falso de aquello de lo que se distancia (Adorno, 2004, p. 236).

Ahora bien, si el aspecto procesual de la obra de arte se codifica en su objetivación, se constituye también en testimonio de su temporalidad: "el carácter procesual de las obras de arte no es otra cosa que su núcleo temporal" (Adorno, 2004, p. 237). En el reconocimiento de su condición temporal la obra es polémica, la obra de arte se defiende del "estatismo atemporal", mediante la afirmación de su "carácter de tensión". Es esta una dimensión más que especifica a la obra como proceso o como ser consciente de su devenir: la obra se instala en la temporalidad con consciencia de su carácter finito. Esta caducidad de la obra hace parte también de su carácter procesual. Y por ello, la obra de arte es menos objeto que experiencia. Ya volveré sobre esto.

En lo que nos interesa, por el momento, es importante habernos apropiado de la denominación de escritura para designar los procesos de configuración de la obra. Debemos solamente avanzar en esa dirección y asimilar el proceso de configuración que es la escritura con los procesos de representación que dan cuenta de la creación de la obra de arte. Aquí debo apoyarme en una discusión que está en "Políticas de la representación", texto que escribí en otro momento (Viviescas, 2017).

Si el aspecto procesual de la obra de arte se codifica en su objetivación, se constituye también en testimonio de su temporalidad: "el carácter procesual de las obras de arte no es otra cosa que su núcleo temporal". 


\section{El arte como representación}

Esta consideración de la obra de arte toma en cuenta el vínculo que la obra de arte establece con lo real; la transformación que el proceso de conformación de la obra de arte opera sobre esto real mediante su postulación o tratamiento como material y de la incorporación de procedimientos y modos de hacer que transforman el material en obra; la condición autónoma de la obra de arte que, en mi lectura, identifica menos su condición alternativa y distinta respecto de lo real, sino que tiene como propósito señalar las condiciones complejas de la relación entre obra de arte -autónoma-y realidad y mundo. Esta reflexión sobre la obra de arte toma en consideración también el efecto o permanencia de la obra en el entramado de la vida -individual, social, pública- en la que continúa el espectador y en la que la obra sigue operando como huella o como efecto.

Por último, pero debe haber sido intuido desde el principio, esta reflexión reconoce la dimensión espiritual de la obra de arte, que adviene, que existe como fenómeno -objeto o acontecimiento - gracias a su capacidad de ser en lo material sensible. En esta consideración coinciden Hegel y Adorno. El segundo solo distanciándose del primero en una limitación de la dimensión idealista de la concepción del primero, respecto de la imposibilidad de una transmutación total de lo material en

\section{La condición autónoma de la obra} de arte que, en mi lectura, identifica menos su condición alternativa y distinta respecto de lo real, sino que tiene como propósito señalar las condiciones complejas de la relación entre obra de arte

-autónoma- y realidad y mundo. espiritual, ya volveremos sobre esto. De allí que una segunda diferencia importante, sea que para Adorno la obra tenga una condición heterogénea -material y espiritual o de arte y no arte - que especificaría a la obra de arte moderna. Condición heterogénea que es fundamental en mi reflexión.

El arte como representación se objetiva en la operación -o en la serie de operaciones - que se representa en la escritura. El arte como escritura supone un trabajo de conformación, de articulación de materiales, en el que el proceso mismo y el tratamiento de los materiales, por efecto de la ley formal, verifican el tránsito de los materiales del mundo empírico en obra de arte $^{1}$. En este sentido, la representación y la escritura como operación que la objetiva son sendos mecanismos de transformación de lo real en escritura, es decir, en obra de arte.

Esta formulación del arte, en tanto aprehensión de lo real, ya como representación o solo como presentación, cruza de manera transversal todas las dimensiones de la creación artística. Pero se detiene de manera especial en la poiesis plástica de los materiales, por su condición de momento en el proceso de la representación. De manera estratégica esta formulación privilegia el momento poiético del proceso, porque es allí donde lo real es apropiado como material, para ser configurado y dispuesto como obra. La detención en la poeisis se justifica porque su devenir tanto como su destino, ya como momento de mediación, ya como fin en sí misma, definen las posibilidades de la representación o del arte del presentar, su sentido y sus políticas. Sobre todo en lo que tiene que ver con la centralidad o la condición liminar de la mímesis como destino final o determinación de la poeisis.

Son materiales los del contenido, también los matéricos, pero también los de los lenguajes del arte: las técnicas, los procedimientos, las operaciones y los dispositivos. 
Y esto es así, porque el pensamiento de las políticas de la representación no hace solamente referencia a los contenidos o sentidos que la obra vehicula o produce. Hace también referencia a las operaciones de conformación a las que se somete este material, y al sentido de estas operaciones, comprendidas como modos de hacer, formas de disposición, técnicas de estructuración. En el entendido de que la poiesis engloba en el mismo gesto la convocación de los materiales, sus procesos de conformación y los procedimientos y los dispositivos a través de los cuales el todo se dispone como artefacto, el artefacto que es la obra de arte, ya sea este objeto o experiencia.

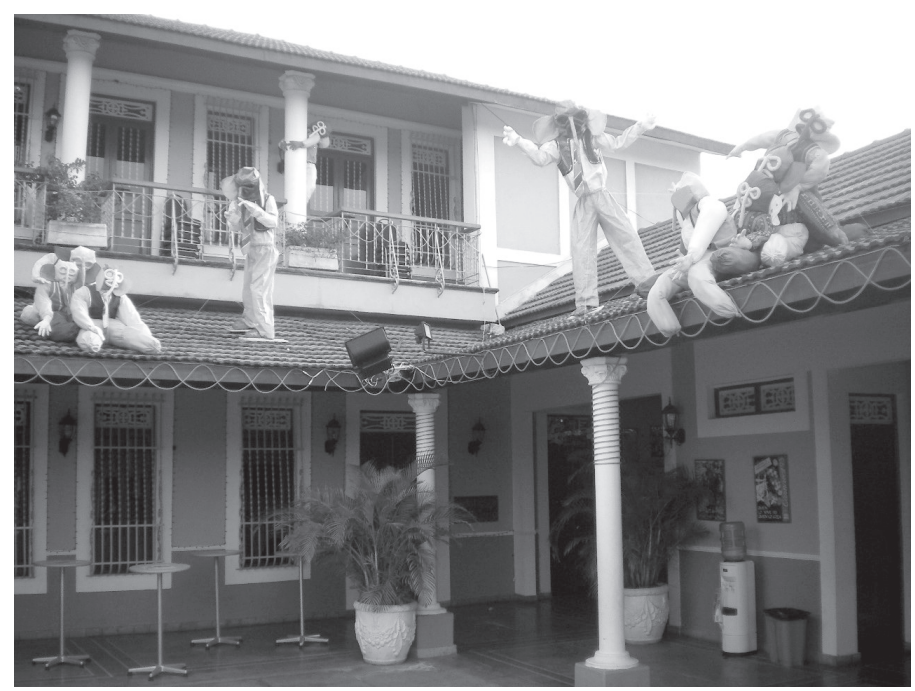

\section{La obra de arte como experiencia}

Estamos ahora en condición de comprender la obra de arte no como un objeto, sino como un proceso hecho de momentos. No como un objeto, sino como experiencia. Y experiencia en un doble sentido. En primer lugar, como acontecimiento o suceso que involucra al espectador-lector para poder devenir como obra. Y, en segundo lugar, como experiencia también que destaca la condición de proceso y de duración que tiene la obra de arte. En la primera acepción es importante comprender que la obra de arte $-\mathrm{y}$ ahora podríamos decir, la obra de arte contemporánea - es menos un objeto y es más la invitación a una experiencia: un suceder en el espacio y el tiempo que afecta al sujeto-espectador al que provoca afectivamente y al que reclama su participación para poder efectuarse como obra de arte. Pero en el segundo sentido, el reconocimiento de la obra de arte como proceso nos permite también reclamar que la obra de arte hace parte de un continuo del que se separa, y de manera sintética, podríamos llamar la historia del arte.

De nuevo estamos en el terreno que marca Theodor Adorno para la obra de arte. En la experiencia de la obra de arte, cada obra reclama su singularidad y la inmediatez del contacto del espectador consigo mismo. Pero esa experiencia estética en Adorno está también complejizada en una dilatación temporal. En una doble metáfora, Adorno expande el momento singular de la obra de arte. En un primer momento, como metáfora temporal, la experiencia estética está constituida de momentos y caracterizada por la duración. En una segunda consideración, ahora como metáfora espacial, la obra de arte está constituida por capas o estratos: la superficie del primer contacto se constituye como umbral que nos invita a trascender el portal y recorrer-reconocer los siguientes estratos que constituyen la obra de arte. En cualquiera de las dos metáforas que definen la experiencia estética, esta es dilatada y compleja, según la consideración de Theodor Adorno.

En lo que a mí respecta, la complejidad de la experiencia estética nos permite desplegar la obra de arte en varios momentos o componentes.

Sí, sin duda la obra de arte se define por su singularidad y su autonomía. Es singular porque es un "uno" que no tiene repetición posible: ni antecedente ni consecuente. Y es autónoma, porque proviene "solo" de la decisión de su autor -individual o colectivo- en el encuentro 
y en la vocación configuradora de y con los materiales. Estas dos condiciones garantizan la unicidad de la obra. $Y$, sin embargo, de manera inmediata, esta unicidad y excepcionalidad de la experiencia estética reclaman la contrastación de esta - de cada- obra particular con la tradición, con la historia del arte. Es por contraste con la historia y la tradición que la singularidad de la obra de arte afirma y garantiza esta singularidad. Es por contraste -memoria y olvido- de los procedimientos y las técnicas de conformación - configuración que las obras de arte reconocen la tradición que continúan o la que trasgreden. De hecho, es por contraste con la tradición y la memoria que la obra de arte $-y$ el artista- establece una tradición a la que reconoce como antecedente de su acción actual. En fin, es por contraste con la tradición, por vocación de continuidad o de ruptura, que la obra de arte $-\mathrm{y}$ los artistas- establecen la potencia de los materiales que toman como materia de la obra de arte (es la tradición la que ilumina la nobleza de materiales como el mármol, la piedra, la arcilla, el vidrio, la basura).

Con esto quiero establecer que la obra de arte es un acontecimiento singular, es un proceso que adviene, que llega a ser, que fructifica, y no solamente un objeto. Que en tanto que proceso, la obra de arte está siempre en tensión con el mundo empírico del que proviene $-\mathrm{y}$ al que abandona en su acaecer- y también en tensión con el mundo del arte del que proviene y al que se adscribe. Esta tensión es al mismo tiempo de reclamo para poder advenir -es una exigencia de existencia- tanto como de censura o superación de lo que la antecede -toda obra de arte reinventa el arte que mejor expresa y, por fuerza, al reconocer una tradición, sanciona como caduca la tradición a la que se inscribe, al menos como pasado de su propio presente.

Si en términos de la historia del arte la tensión que caracteriza el advenimiento de la obra de arte, que nombramos en segundo lugar, inaugura o nombra el campo de la historia del arte como necesario en la formación de artistas, la tensión que nombramos en primer lugar inaugura o da ingreso al efecto que la obra de arte produce. Este efecto es de producción de un conocimiento a otro. $\mathrm{Y}$ esto tanto en la comprensión de la estética de Hegel como en la comprensión de Theodor Adorno.

\section{Mimesis y representación en $\mathrm{Hegel}^{2}$}

En las Lecciones de Estética (Hegel, 2007) Hegel postula la superioridad del arte sobre la naturaleza, como condición específica de lo que denomina lo "bello artístico". Habiendo establecido la superioridad de lo "bello artístico" sobre lo "bello natural", Hegel acomete la demostración de que esto bello artístico no puede quedarse en mero concepto general, sino que "debe pasar a la determinación real"; es decir, que lo bello artístico, según su concepto mismo, "tiene en sí determinación y particularidad". Esta salida de sí de lo bello artístico hacia la determinación y la particularidad obliga su salida a la finitud y a la exterioridad,

2 De nuevo, cfr.: Víctor Viviescas (2017) "Políticas de la representación" (en imprenta). en esta dialéctica específicamente hegeliana que reclama que la "idea", para devenir verdad, "tiene que pasar a la realidad".

En el caso de las obras de representación -narrativa y dramaturgia - este devenir, este pasar a la realidad compromete dos niveles: el nivel más superficial de la lengua y el nivel del lenguaje de estructuración genérica de la escritura. En la estructuración genérica, la acción es determinante y prioritaria en la representación. Este "pasar a la realidad", de la idea, es justamente lo que podemos llamar representación. El sentido de la acción como origen de la representación es el de privilegiar la condición mimética de la obra. La interpretación de este pasar a la realidad mediante la 
mímesis, las consecuencias que se desprenden de él, constituyen su dimensión política (Hegel, 2007).

Hegel establece una relación entre la representación interna y subjetiva de las diferentes artes y la representación objetiva, es decir, los medios, los artefactos, los dispositivos mediante los cuales se objetiva esta representación. En el caso de la literatura, que cae en la órbita de lo que denomina la poesía, como arte que es de la palabra, Hegel la identifica como el tercer elemento o la síntesis de las artes "objetivas", en las que prima el dispositivo externo -como la pintura- y las artes "subjetivas", donde prima la interioridad del ejecutante -como la música-, es decir, la síntesis, "la totalidad", que "unifica en sí los extremos de las artes figurativas y de la música en una fase superior":

El dominio de la representación [subjetiva o íntima], que no se queda en la interioridad más abstracta como tal, sino que se configura su mundo como una realidad efectiva concreta, se separa por su parte igualmente de la música y se da para sí en la poesía una existencia conforme al arte" (Hegel, 2007, p. 696).

La representación poética se diferencia de la representación prosaica por el contenido, por la modalidad misma de la representación y por la particularización de la intuición poética.

El problema del conocimiento, como efecto de la obra de arte, se comprende al saber que, como contenido, el arte no tiene las cosas naturales o contingentes sino lo que Hegel llama "los intereses espirituales", es decir, que el objeto del arte es "el infinito reino del espíritu" (Hegel, 2007, p. 704).Todo el círculo de lo sensible, de lo intuitivo, de lo material, entra a la poesía solo en la medida en que en él encuentra el espíritu un estímulo o un material para su actividad: como entorno del hombre, como su mundo exterior que "sólo tiene un valor esencial en la relación con lo interno de la consciencia, pero que no puede aspirar a la

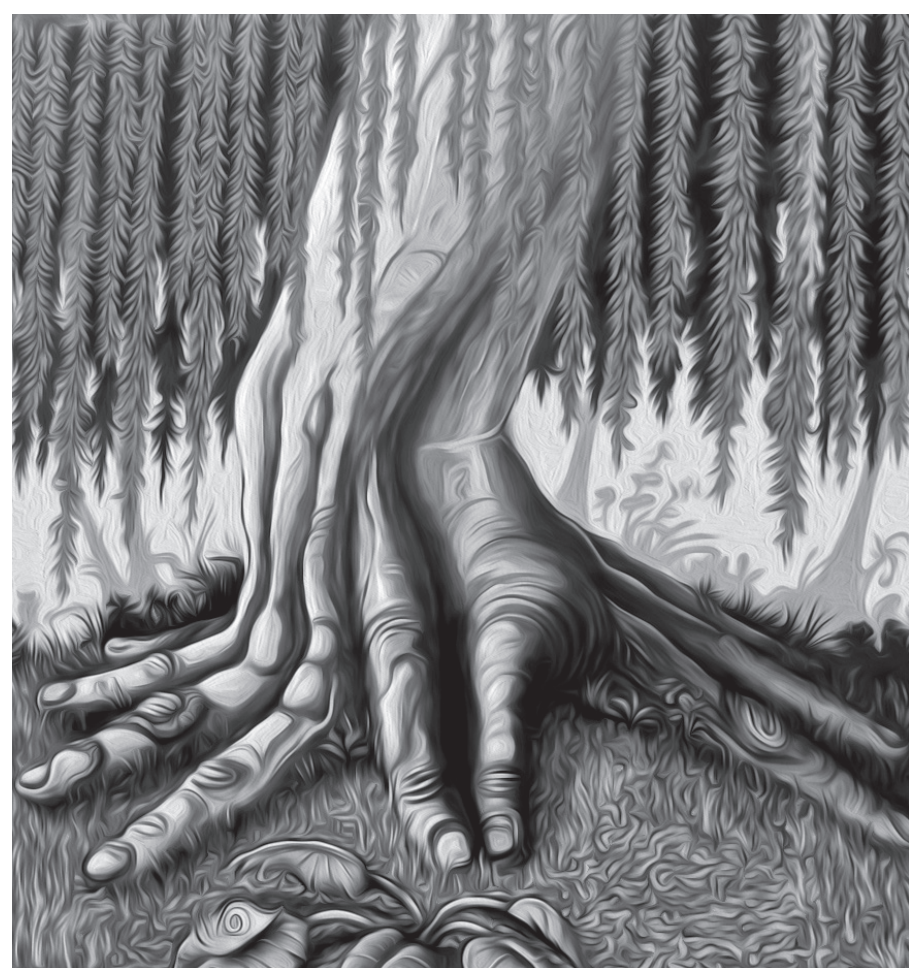

dignidad de devenir para sí mismo el objeto exclusivo de la poesía" (Hegel, 2007, p. 704).

La condición que especifica a la representación poética y que la diferencia de la representación prosaica es que es "representación originaria de lo verdadero". Es decir, la poesía es un saber que "todavía no separa lo universal de su existencia viva en lo singular" (Hegel, 2007, p. 704). Es decir, que "en la poesía lo universal, lo racional, no es expresado en universalidad abstracta y conexión filosóficamente demostrada, o con referencia intelectiva de sus aspectos, sino como vivificado, aparente, animado" (Hegel, 2007, p. 705).

Finalmente, la representación poética procede mediante una particularización aun mayor que la de las demás artes que tienen un desarrollo menos rico, porque, según Hegel, la poesía "abarca el espíritu del hombre en su integridad y la humanidad está múltiplemente particularizada" (Hegel, 2007, p. 707), lo que lleva a identificar y diferenciar las poesías nacionales $y$ aun las de los diferentes periodos. 


\section{Pensamiento de la obra de arte en Adorno}

El efecto final de la obra de arte es para Theodor Adorno un efecto de conocimiento. Es conocimiento y al mismo tiempo es inefable. La explicación posible es que este es un conocimiento otro: no discurso, no del concepto, es decir, no del logos. Pero sí contundente y definitivo como la transformación. Adorno lo va a vincular a una iluminación mesiánica, en la que no tengo el tiempo de detenerme.

La obra de arte tiene un efecto demoledor y contundente en el espectador. Este efecto es interpretado por Adorno como conocimiento. Sin embargo, en la crítica a la razón ilustrada, Adorno preserva al arte del poder dominador del pensamiento de lo idéntico que se objetiva en el discurso conceptual y en el conocimiento lógico-procedimental. De manera sintética, Adorno dice que la obra de arte preserva el círculo del mito que ha sido destruido por la razón ilustrada. Esta filiación de la obra de arte con el mito y con lo mágico determina desde entonces su condición respecto del saber lógico: que se funda en el discurso lógico y, en definitiva, en el concepto.
Podemos entonces reconocer una especie de dimensión epistemológica de la experiencia estética, pero a condición de reconocer siempre que nos movemos en un terreno metafórico: que la sabiduría que el arte produce como conocimiento no se confunde con el saber discursivo del conocimiento lógico-racional.

Pero debemos también volver un poco a un argumento ya exhibido, que es el del reclamo de un conocimiento del arte para poder reconocer la singularidad de la obra de arte actual. Adorno se apoya en su comprensión diacrónica de la experiencia estética para reclamar un encuentro libre de prejuicios en la obra de arte, en un primer momento, pero que debe completarse con una vinculación y contrastación de la obra de arte singular con la obra de arte en la historia. De manera paradójica, podríamos decir que la experiencia estética es una sucesión de olvidos y memorias que actúan como un fluido o una experiencia de contrastes en el encuentro con la obra de arte.

\section{En la producción de la obra de arte hay entonces}

momentos de investigación-indagación-intuición y momentos

de conformación-estructuración que suelen ser subsumidos bajo

\section{la denominación de creación.}

\section{Investigación creación}

La discusión de investigación-creación es menos una pregunta del arte y de los artistas y es, más que nada, una pregunta de tipo institucional, en la que los profesores y funcionarios universitarios se preguntan -nos preguntamos- cómo hacer parte de las comunidades académicas de las universidades -con una equivalencia a la pregunta de cómo ser parte de una comunidad científica para participar de las dinámicas de promoción de la ciencia y, por tanto, también del arte-. Es decir, esta es una pregunta más gremial e institucional, que tiene una dimensión sin duda importante en los procesos de institucionalización de las comunidades científicas y en la definición de políticas de fomento y estímulo de estas actividades y comunidades. Por eso podemos llegar a ella con cierta morosidad.

En el recuento por la reflexión estética de Hegel, en la consideración de la obra de representación poética, y de Theodor Adorno, en la 
comprensión de la obra como escritura y de su dialéctica de relación con la realidad, hemos podido verificar, mediante la dimensión espiritual del arte, que la obra de arte, es decir, la experiencia estética produce conocimiento. La obra de arte proviene del mundo de lo real, pero lo transforma en el proceso de su constitución. Esta transformación se da tanto en el proceso compositivo como en el momento de la recepción por parte del lector-espectadoroyente: la obra de arte transforma el mundo. Incluso, podríamos decir, cuando vuelve a mostrarlo para que lo veamos con otros ojos, con otra mirada.

En la producción de la obra de arte hay entonces momentos de investigaciónindagación-intuición y momentos de conformación-estructuración que suelen ser subsumidos bajo la denominación de creación. Si la conformación de la obra de arte es denominada como creación- a pesar de su última referencia a un dios demiurgo-, fuerza es reconocer que esta operación incluye momentos dispares, diferentes, que es una actividad heterogénea. Dentro de esta heterogeneidad ocupa un lugar no menor el momento formal, que pasa por la apropiación, manejo y transformación de los medios, las técnicas y los lenguajes, que son los que configuran, los que, estrictamente, dan forma.

La obra de arte, entonces, participa de procesos de investigación para su producción, es decir, para su creación. Tanto como produce conocimiento en su creación y en la experiencia estética que produce. Esta doble condición la hace un objeto factible de procesos de investigación-creación.

Una digresión sobre el campo teatral puede ilustrar mejor mi pensamiento. En la tradición del teatro europeo contemporáneo de mitad del siglo XX fue fundamental el surgimiento de un teatro laboratorio o un teatro experimental que aparecía para constituirse en una tercera vía respecto del teatro comercial y del teatro de arte, que existían de manera estable en la tradición de las principales capitales de Europa, desde Moscú hasta Londres, pasando por Berlín y París, pero yendo también a ciudades pequeñas como Wroclaw en Polonia, donde se asienta en 1965 el Teatro Laboratorio de Jerzy Grotowski. La denominación "teatro laboratorio" designaba entonces $-\mathrm{y}$ a partir de entonces- un trabajo teatral de búsqueda, experimentación y sistematización de nuevas posibilidades de la creación teatral, que iban desde la transformación de los lenguajes, la modificación de la dialéctica entre gesto corporal y palabra, hasta al sentido espiritual de la obra de arte como proceso de transformación, en primer lugar, del actor mismo. En una gran medida, el teatro moderno en Colombia surge y se consolida como teatro experimental, en la constelación de sentido que le dan experiencias como la liderada por Grotowski, pero también por Peter Brook en Londres y luego en París, Eugenio Barba en Holstebro, pero también Arianne Mnouchkine, de nuevo en París. El teatro laboratorio, tanto como el teatro experimental, se instalan en un lugar de investigación- experimentación que dota de un sentido distinto a la creación teatral, como resultado de un proceso de interrogación de las convenciones teatrales existentes. Podríamos decir de manera más amplia, como resultado de un conocimiento e interrogación de la tradición teatral.

Tal como podemos verlo en la digresión sobre el campo teatral, también el campo amplio de la creación tiene distintas modalidades o diversos énfasis en una actividad que suele ser tomada como única, según esta denominación de creación. Aquí, en este punto, el énfasis que quiero establecer es que la creación puede proponerse como proceso de investigaciónexperimentación, distinto respecto de la creación misma. Por otra parte, tal como vimos antes, la obra de arte es el resultado de un proceso de creación que no puede ser confundido con ningún otro proceso de apropiación y transformación de la realidad. De manera precisa, no puede ser confundido con 
las prácticas de construcción de conocimiento discursivo de tipo lógico-instrumental, pero tampoco por procedimientos de apropiación y transformación de la naturaleza para su disposición de uso utilitario, como hacen la técnica y la tecnología. Y en este punto, mi énfasis es en la condición de producción de conocimiento otro que tiene la obra de arte.

Redundemos sobre esto, es decir, sobre la posibilidad de que la obra de arte en su proceso de constitución pueda ser objeto de un trabajo de investigación creación. La obra de creación artística y el proceso mediante el que surge son ya procesos de producción de conocimiento. La obra de investigación-creación suele, además de lo que es propio de la creación mencionada, proponerse preguntas o interrogantes que operan de una manera distinta a como operan en el campo de las ciencias y la tecnología, pero que actúan como orientadores de un proceso que debe poder contar con el azar, con lo imprevisto, con la intuición y la propuesta heurística. La denominación investigacióncreación sigue nombrando una actividad y un modo de actuar en el conocimiento, apropiación y transformación de los modos de hacer de la obra de arte que sigue estando vigente y sigue aportando singularidad al trabajo de investigación y experimentación en el campo de la creación.

Finalmente, es importante volver a poner en contexto esta discusión. La pregunta sobre la investigación-creación en Colombia en los últimos años, se plantea en el espacio institucional de la universidad. La denominación investigación-creación surgió hace alrededor de 30 años en el marco de la discusión y el reclamo por el trato igualitario de los artistas en el marco de las universidades y en el campo de la investigación científica que administra el instituto de fomento a la ciencia (Colciencias). En una gran medida intentaba responder al interrogante: ¿cómo hacer un reconocimiento a la producción de creación por parte de los artistas vinculados al campo académico, que equivaliera al reconocimiento de que son objeto las producciones de investigadores científicos, sociales y de la técnica y la tecnología?

La universidad, como constructo institucional y disciplinar, supone la coexistencia en comunidad de estos diferentes saberes. En la configuración de la universidad moderna en el siglo XIX, el último de los saberes integrados es el del arte. Pero es en ese contexto que cobra especial relevancia la pregunta por la asimilación o correspondencia entre creación e investigación.

La discusión sobre la autonomía y relevancia de la obra de arte como creación solo se puede comprender en el marco de esta comunidad -imaginaria- que se constituye en la universidad. En ese contexto, más que crear disyuntivas, de lo que se trata es de identificar los campos de posibilidad que abre cada una de estas modalidades de actividad y de acción. De manera especial, los campos que pueden ser compartidos. La construcción de comunidad tiene que ver con la capacidad de construir $-\mathrm{y}$ defender- singularidades. Pero también tiene que ver con la posibilidad de establecer diálogos e interacciones. ¿Cuál es el proyecto de universidad que nos estamos proponiendo? Es la respuesta a esta pregunta la que abre las condiciones de posibilidad de las discusiones sobre investigación y creación.

\section{Referencias}

Adorno, T. W. (2004). Teoría estética. Madrid: Akal.
Viviescas, V. (2017). Políticas de la representación (en imprenta).

Hegel, G. W. F. (2007). Lecciones sobre la estética. Madrid: Akal. 